\title{
Bilateral Thalamic Tumour in Children: Therapeutic Problems in Our Setting - A Case Report and Review of the Literature
}

\section{Mhacks Malangu, Elisée job Befio, Jean Michel Nzisabira, Célèbre Mualaba*, Daddy Singoto and Momar Codé Ba}

\section{Department of Neurosurgery, Fann Teaching Hospital, Dakar, Senegal}

*Corresponding Author: Célèbre Mualaba, Department of Neurosurgery, Fann

Teaching Hospital, Dakar, Senegal.
Received: July 16, 2021

Published: July 29, 2021

(C) All rights are reserved by Célèbre

Mualaba., et al.

\section{Abstract}

Thalamic tumours in children are rare, although their incidence has not yet been established. We report a case found in our milieu in order to share experience and awake practitioner about the existence of this lesion in children.

Keywords: Thalamic Tumour; Therapeutic Problems; Children

\section{Introduction}

Thalamic tumours in children are rare, although their incidence has not yet been established. two recent reports provide figures ranging from $0.84 \%$ to $5.2 \%$ of all intracranial tumours. this wide range of incidence is mainly related to the difficulty in differentiating primary thalamic tumours from those lesions that secondarily involve thalamic structures, originating mainly in the cerebral hemispheres, caudate nuclei, pineal gland and brainstem. secondary invasion of both thalamic nuclei is not uncommon, especially in the late stages of the progression of the neoplastic process, and the phenomenon can be observed in up to one third of patients. on the other hand, primary bilateral thalamic tumours (pttb) are considerably less frequent; their description is limited to anecdotal reports of single cases, both in adult and paediatric populations [1].

\section{Case Observation}

A 9-month-old patient with no previous history who consulted for the management of recurrent vomiting and whose examination revealed axial hypotonia. Medical treatment was instituted.
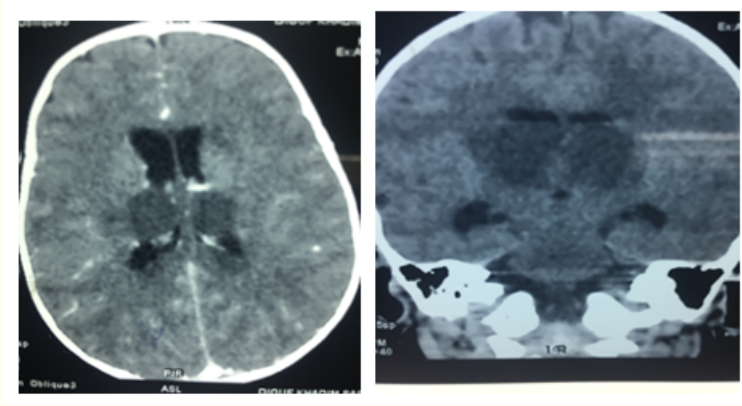

Figure 1: CT SCAN: appearance in favour of bilateral thalamic hypodense patch, not enhancing after injection of contrast medium.

\section{Discussion}

Primary thalamic tumours account for only 1 to $1.5 \%$ of all intracranial tumours and about $25 \%$ of them occur in children aged 15 years or younger. However, their true incidence has not yet been established and two recent reports provide figures ranging from 0.84 to $5.2 \%$ of all intracranial tumours [9]. 


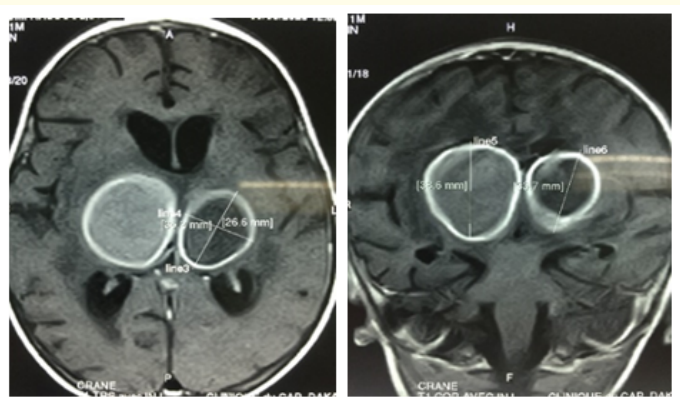

Figure 2: MRI: bi-thalamic lesion formation, partially haemorrhagic, intense annular enhancement, with mass effect on V3 and upstream dilatation.

The clinical picture of bilateral paediatric thalamic tumours is variable. It includes: intracranial hypertension syndrome, hemiparesis, sensory disturbances, tremor, dysmetria and unsteady gait may be present $[1-3,6,7,9]$.

MRI has greatly improved the possibility of surgical resection of thalamic tumours, demonstrating their precise localisation and accessibility and allowing accurate postoperative evaluation of surgical resection [1-7,9,10]. Bilateral thalamic tumours were predominantly heterogeneous on T2 MRI [11]. In our series, brain MRI and brain CT were performed.

From a therapeutic point of view, the management is multidisciplinary (neurosurgeons, oncologists and radiotherapists) and the diffuse and bilateral involvement of the thalamic nucleus by the tumour makes surgical therapy very problematic, even today. Neuronavigation and stereotactic surgery are still limited by the size and topography of these tumours. Theoretically, radical excision may be possible only for the most benign and noninfiltrative neoplasms, those that displace rather than infiltrate the cerebral parenchyma, such as gangliocytomas or most juvenile pilocytic astrocytomas, but, to our knowledge, none of the cases have been described in the literature [1,3]. Neuronavigation and intraoperative monitoring of motor and somatosensory evoked potentials were systematically used [3]. The main role of surgery is always to obtain a diagnostic histological examination [1]. In our series, only medical treatment was instituted given the surgical complexity especially in our setting.
From a surgical point of view, thalamic tumours are divided into three types [13]:

- Pure thalamic tumour: those that originate in the thalamus and wish to leave it.

- Thalamo-peduncular tumours: those that occur in relation to these 2 structures

- Primary bilateral thalamic tumour.

The choice of the approach depends on the location of the lesion in the thalamus and its relationship to the surrounding structures. There are several surgical approaches for the removal of bilateral thalamic tumours, especially for unilateral forms $[1-4,10]$ :

- The trans cortical, trans ventricular route

- The anterior or posterior inter-hemispheric pathway

- The supra cerebellar infra tentorial pathway

- The trans-temporal route.

The management of hydrocephalus requires in some cases a cerebrospinal fluid shunt (ventriculoperitoneal shunt, external shunt, endoscopic ventriculocysternostomy, etc.) $[3,6,8,10]$. No CSF shunts were performed in our series.

Radiotherapy and chemotherapy are sometimes used as adjuvant treatment, but their role is debatable [1,4,6,8,10,11]. Their indications depend on histological findings, clinical status, and postoperative imaging [3].

\section{Conclusion}

Bilateral thalamic tumours in children are rare and their clinical presentation is variable. Cerebral MRI is the imaging examination par excellence and their management is multidisciplinary, a real surgical challenge especially in our environment.

\section{Bibliography}

1. Concezio DR and Aldo I. "Bilateral thalamic tumors in children". Child's Nervous System 18 (2002): 440-444.

2. Ulaş C., et al. "Bilateral thalamic gliomas: Report of two cases with review of literature". Neurology India 62.4 (2014): 444446.

3. Giuseppe C., et al. "Surgical treatment of thalamic tumors in children". Journal of Neurosurgery Pediatrics 21 (2018): 247257. 
4. Dinesh K Rajput., et al. "Bilateral thalamic glioma in a 6-yearold child". Journal of Pediatric Neurosciences 5.1 (2012): 45-48.

5. Hirano S., et al. "Bilateral thalamic glioma: case report". Neuroradiology 42.10 (2002): 732-734.

6. Stephanie P., et al. "Thalamic tumors in children: a reappraisal". Journal of Neurosurgery 106 (2007): 354-362.

7. Eranga G., et al. "A case report on 1-year follow-up of bilateral thalamic glioma". Neuroradiology Journal 28.6 (2015): 584586.

8. Xiaodong N., et al. "Prognostic Factors for the Survival Outcome of Bilateral Thalamic Glioma: An Integrated Survival Analysis". World Neurosurgery 110 (2018): e222-e230.

9. Girish M., et al. "Bilateral thalamic glioma: Report of four cases and review of literature". Journal of Pediatric Neuroscience 1.2 (2006): 66-69.

10. Marc B., et al. "Surgical resection of thalamic tumors in children: Approaches and clinical results". Child's Nervous System 23 (2007): 753-760.

11. Ryan PL., et al. "Surgical and molecular considerations in the treatment of pediatric thalamo peduncular tumors". Journal of Neurosurgery Pediatrics 20 (2017): 247-255.

12. Paul S., et al. "Pediatric thalamic tumors in the MRI era: A Canadian perspective". Child's Nervous System 32 (2016): 269-280.

13. Christian SR., et al. "Thalamic Glioma. Neuro-Oncology of CNS Tumors" 363-375.

\section{Volume 4 Issue 8 August 2021}

\section{(c) All rights are reserved by Célèbre Mualaba., et al.}

